\title{
Structural changes of tissue samples exposed to low frequency electromagnetic field: A FT-IR absorbance study
}

\author{
V. Crupi ${ }^{\text {a,* }}$, S. Interdonato a ${ }^{\text {, D. Majolino }}{ }^{\text {a }}$, M.R. Mondello ${ }^{\mathrm{b}}$, S. Pergolizzi $^{\mathrm{c}}$ and V. Venuti ${ }^{\mathrm{a}}$ \\ a Dipartimento di Fisica dell'Università di Messina \& INFM Sezione di Messina, \\ C.da Papardo S.ta Sperone 31, 98166 Messina, Italy \\ ${ }^{\mathrm{b}}$ Dipartimento Farmaco-Biologico, Università di Messina, SS. Annunziata, Messina, Italy \\ c Dipartimento di Biomorfologia dell'Università di Messina, C.da Papardo S.ta Sperone 31, \\ 98166 Messina, Italy
}

\begin{abstract}
In the present work, we report on a preliminary Fourier Transform Infrared (FT-IR) Absorbance study performed on different kind of rat tissues, such as kidney and heart, exposed to a "non-ionizing" radiation source at low frequency, in the range typical of micro-waves $(300 \mathrm{MHz}<\nu<300 \mathrm{GHz})$. The data were collected in a wide wavenumber region, from $400 \mathrm{~cm}^{-1}$ to $4000 \mathrm{~cm}^{-1}$. The comparison of the absorbance spectra in the case of the normal tissues with the irradiated ones has shown significant differences in the spectral features in accordance with the morphological analysis performed by the optical microscopy.
\end{abstract}

Keywords: Infrared spectra, electromagnetic field, vibrational dynamics

\section{Introduction}

In the last years the international scientific community has devoted an increasing interest in the study of the environmental and health impact related to the use of devices that irradiate electromagnetic fields, EMF [1]. In particular, a great attention has been payed to the observation of the dangerous effects on the health caused by "non-ionizing" radiation at low frequency, in the range typical of radio-waves $(\nu<300 \mathrm{MHz})$ and micro-waves $(300 \mathrm{MHz}<\nu<300 \mathrm{GHz})$.

Generally speaking the cellular responses to the various forms of the radiation, including ionizingand UV-irradiation or exposure to electromagnetic fields is manifested as irreversible and reversible structural and functional changes to cells and cell organelles. Moreover, beside the morphological signs related to the cell death, there are several reversible alterations in the structure of different cell organelles. So that it is worth stressing that the biological effects related to the exposure of EMF are not always indication of pathologies, dangerous alterations and modifications for the human health. An example of a huge controversy in this field is given by the eventual dangerous effects due to the mobile phones. In fact these last emit radio-frequency energy at levels high enough to reach base stations (antenna towers)

\footnotetext{
${ }^{*}$ Corresponding author: Dr. Vincenza Crupi, Dipartimento di Fisica, Messina University, PO Box 55, Italy. Tel.: +39 090 6765447; Fax: +39 090 395004; E-mail: vcrupi@unime.it.
} 
tens kilometres away. Since the energy is emitted as a micro-wave in the direct vicinity of the users head there are concerns about the safety of this technology. And speaking about this there are reports from cell studies as well as animal studies that are most worrying. There are few illnesses and ailments that have potential links to cell-phone radiation: cancer, brain tumors, headaches and fatigue. To effect a change in biological material through which it is passing, an EM wave must deposit enough energy to alter some structure significantly. But every material particle within the body already possesses an average thermal kinetic energy of the order of kT and these particles continually collide with other particles of similar energy. For a change to occur in biological material, the EM wave seemingly should transfer energy considerably above kT to selected particles, and at $T=310 \mathrm{~K}\left(37^{\circ} \mathrm{C}\right.$, body temperature $)$ $\mathrm{kT}=4.3 \times 10^{-21} \mathrm{~J}$. Another standard of comparison is the chemical bond, because to be effective in promoting change the field should be able to deposit packets of energy larger than the bond energy, and bonds are typically within an order of magnitude of an electron volt $\left(1.6 \times 10^{-19} \mathrm{~J}\right)$. The energy carried by an EM photon is precisely $h f$ where $h$ is the Planck constant and $f$ is the frequency of the wave in Hz. Thus in the range from 300 to $3000 \mathrm{MHz}$, which includes also the RF, the energy is less than $0.1 \%$ of either kT or the bond energy. So that one could argue that there is little prospect of RF irradiation having biological activity at sub-thermal power level. But the global debates and controversy over the health effects of the electronic devices which emit in the range of RF continue. In fact as with most controversial topics, different studies have different results. Some state that cell phones are linked to higher occurrences of cancer and other ailments, while other studies report that cell-phone users have no higher rate of cancer than the population as a whole. Actually no study has still provided conclusive evidence that cell phones can cause any of these illnesses.

Nowadays many different and complementary experimental techniques are addressed to study the eventual dangerous effects of the exposure to EMF on the human tissues. Among the advanced spectroscopic methods, Fourier Transform Infrared (FT-IR) Absorbance represents a good tool in this kind of research $[2,3]$ even from a diagnostic point of view, providing information concerning structure and interactions of proteins, lipids, nucleic acids and so on, in isolation and in complex assemblies. Furthermore, FT-IR spectroscopy is able to detect biochemical changes caused by pathologies [4,5], also at very early stage of the disease, due to the fact that any change in the biochemistry tissue must precede any morphological manifestation of the disease in itself. Finally, it gives the opportunity to analyse, in nearly real time, samples easily prepared, with small size (less than $1 \mathrm{~mm}$ thick and less than $1 \mathrm{~cm}$ in diameter) and furnishes reliable and reproducible spectral data [6].

In the present work, we report on a FT-IR Absorbance study performed on different kind of rat tissues, such as kidney, liver and heart, exposed to a $900 \mathrm{MHz}$ radiation source in order to study eventual structural changes. The data were collected in the wavenumber region from $400 \mathrm{~cm}^{-1}$ to $4000 \mathrm{~cm}^{-1}$. The comparison of the spectral features among the normal tissues and the irradiated ones has shown significant differences in agreement with the morphological analysis performed by the optical microscopy.

\section{Materials and methods}

For the spectroscopic analysis on kidney and heart tissues, we used male Wistar Hannover rats, 250$300 \mathrm{~g}$ weight, kept under standard conditions, with free access to food and tap water. The animals were exposed to a $900 \mathrm{MHz}$ EMF radiation source for $24 \mathrm{~h}$ for 3 months and kept at a distance of $15 \mathrm{~cm}$ from the source inside plastic cages (no metal part). They were sacrificed by cervical dislocation under ether anaesthesia [7]. The tissues were quickly collected from the kidney and the heart and immediately 
frozen in liquid nitrogen for the FT-IR measurements. By using a standard cryostatic methodology with a freezer microtome (Reichert Jung), we obtained thin slides of tissue, about $8 \mathrm{~mm}$ thick, without any embedding procedure [8]. Thanks to this method we were sure to prevent any unwanted contribution in the investigated spectral range, due to the presence of substances eventually used for the fixing procedure. The layers were put on $\mathrm{KBr}$ pellets which were transparent in the analysed wavenumber range, region from $400 \mathrm{~cm}^{-1}$ to $4000 \mathrm{~cm}^{1}$.

The IR absorbance measurements were performed in dry atmosphere and each measurement was repeated several times to be sure of the reproducibility of the spectra. The data were collected on rat kidney and heart tissues, by a FT-IR BOMEM DA8 spectrometer equipped with a globar lamp as source, a $\mathrm{KBr}$ beamsplitter and finally with a DTGS/KBr detector, in order to work in the above mentioned spectral region.

In order to study the structural changes induced by the radiation exposure, we compared the IR absorbance spectra of the control samples of kidney and heart tissues to those of samples exposed to the EMF. In particular, inside the analysed spectral range, we focused our attention on the region extending from $900 \mathrm{~cm}^{-1}$ to $1800 \mathrm{~cm}^{-1}$, that includes the $\mathrm{CH}_{2}$ wagging and $\mathrm{C}-\mathrm{O}$ stretching of collagene, since this last showed the most significant variations. To get a good signal-to-noise ratio, and in order to avoid any unwanted contribution, we worked in dry atmosphere and we used a resolution of $4 \mathrm{~cm}^{-1}$, automatically adding 100 repetitive scans for each run.

\section{Results and discussion}

\subsection{Damage due to EMF exposure on kidney tissues}

Previous optical microscopy measurements on normal and irradiated rat kidney tissues unambiguously showed the increasing of the connective tissue [9]. The kidney disease involved both microvascular glomerural network and interstitium. In particular, the glomerural damage was shown to be given by an increasing of the perivascular connective and the glomerural sclerosis.

Taking these morphological results into account, the tissues have been analysed by FT-IR spectroscopy, in order to put into evidence the effectiveness of this technique in the complex field of biophysics and biomedical science.

Figure 1 shows the FT-IR Absorbance experimental spectra of a control kidney sample and a kidney sample after a three months exposure to $900 \mathrm{MHz}$ radiation, in the $900-1800 \mathrm{~cm}^{-1}$ range including the $\mathrm{CH}_{2}$ wagging and the $\mathrm{C}-\mathrm{O}$ stretching bands of collagene. The shoulder in the band of the control sample centred at $\sim 1374 \mathrm{~cm}^{-1}$ becomes a clearly evident narrow peak at $\sim 1350 \mathrm{~cm}^{-1}$ in the spectrum of the irradiated one.

Changes have been observed also in the peaks of the control sample spectrum centred at $\sim 1562 \mathrm{~cm}^{-1}$ and $\sim 1650 \mathrm{~cm}^{-1}$, linked to the $\mathrm{C}=\mathrm{O}$ stretching characteristic of amide I macromolecule: in place of the two narrow peaks typical of the control sample, a large band is revealed for the exposed tissues, as a consequence of the disappearance of the $\sim 1562 \mathrm{~cm}^{-1}$ peak and the broadening of the one at $\sim 1650 \mathrm{~cm}^{-1}$.

In Fig. 2 we report the FT-IR Absorbance experimental spectra, on the same samples, in the 2500$3700 \mathrm{~cm}^{-1}$ range, covering the $\mathrm{CH}_{2}$ and $\mathrm{CH}_{3}$ stretching vibrations $\left(2500-3000 \mathrm{~cm}^{-1}\right)$ as well as the $\mathrm{O}-\mathrm{H}$ and $\mathrm{N}-\mathrm{H}$ stretching $\left(3000-3600 \mathrm{~cm}^{-1}\right)$ ones. As it can been seen from an inspection of the figure, it seems that the exposure to EMF did not cause any relevant change in this spectral region. 


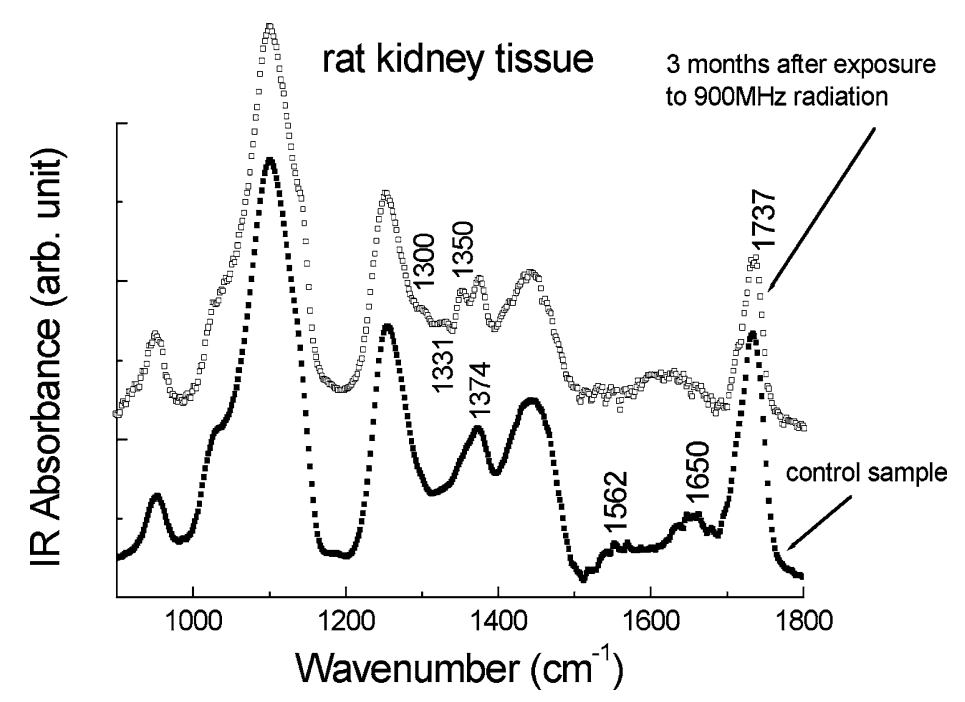

Fig. 1. FT-IR Absorbance experimental spectra, in the $900-1800 \mathrm{~cm}^{-1}$ region, for control kidney sample (closed squares) and kidney sample after three months exposure to $900 \mathrm{MHz}$ electromagnetic radiation (open squares).

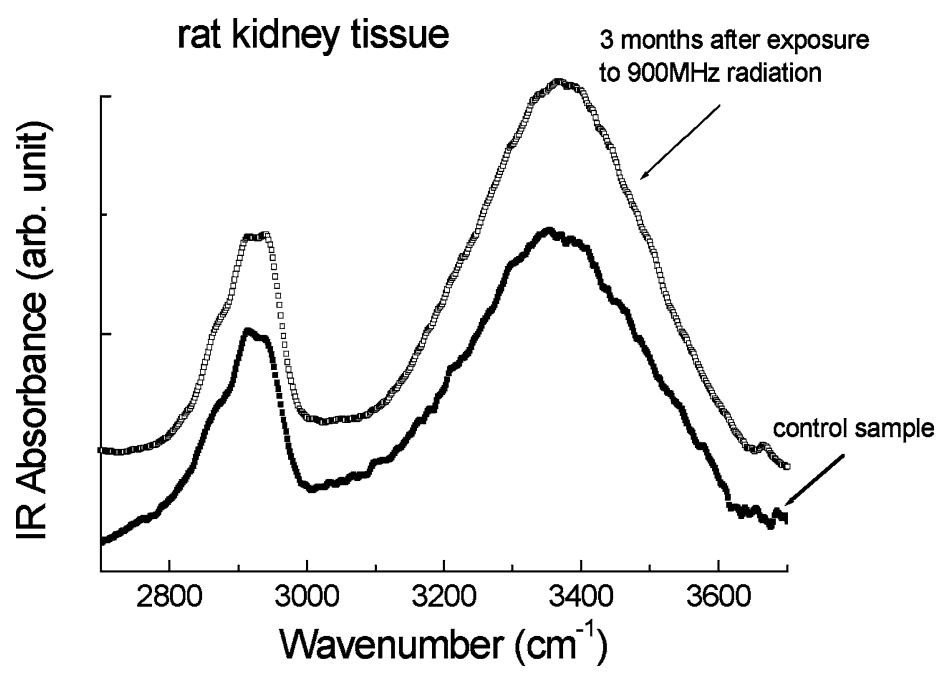

Fig. 2. FT-IR Absorbance experimental spectra, in the $2700-3700 \mathrm{~cm}^{-1}$ region, for control kidney sample (closed squares) and kidney sample after three months exposure to $900 \mathrm{MHz}$ electromagnetic radiation (open squares).

\subsection{Damage due to EMF exposure on heart tissues}

In Fig. 3 the FT-IR spectra in the $900-1800 \mathrm{~cm}^{-1}$ region of a left ventricle tissue for a control heart sample and a sample after three month exposure to $900 \mathrm{MHz}$ EMF are plotted. First of all, we put the attention on the band centred at $\sim 1466 \mathrm{~cm}^{-1}$ and due to the $\mathrm{CH}_{2}$ wagging. Its intensity increases passing from the control sample to the irradiated one. Changes in shape and intensity have been observed also for the peak centred at $1530 \mathrm{~cm}^{-1}$, that reflects a convolution between the $\mathrm{C}=\mathrm{O}$ and the $\mathrm{N}-\mathrm{H}$ stretching typical of amide II macromolecule. Going on, the double-peaked band at $1650 \mathrm{~cm}^{-1}$, corresponding to the $\mathrm{C}=\mathrm{O}$ stretching of amide $\mathrm{I}$ of the control sample, appears to be a large non-structured band in the spectrum of the exposed heart tissue. 


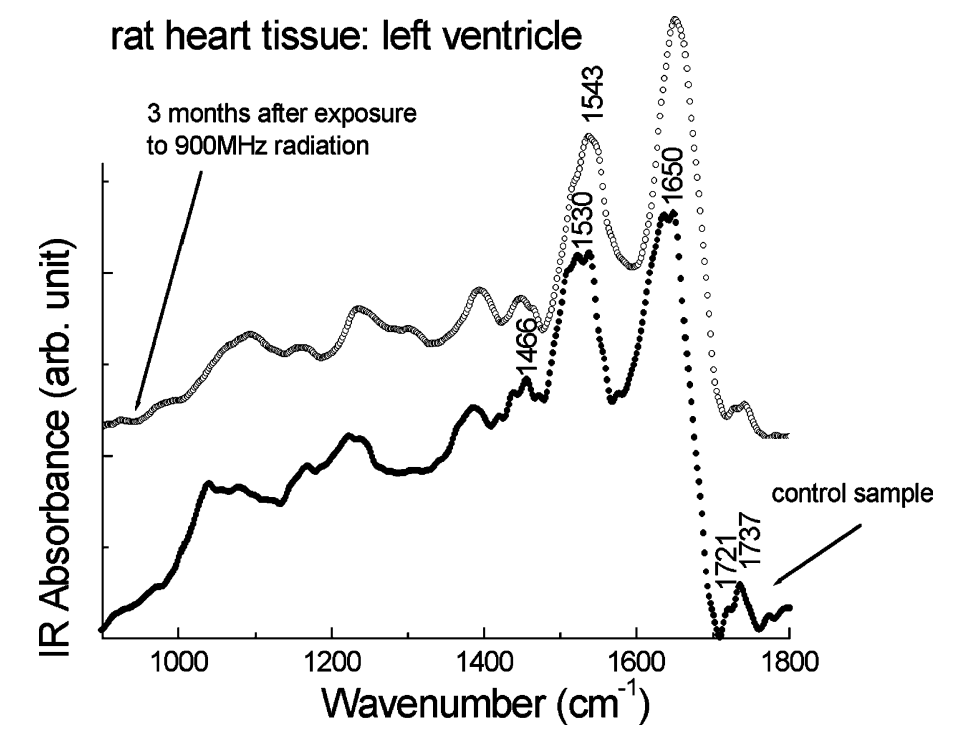

Fig. 3. FT-IR Absorbance experimental spectra, in the $900-1800 \mathrm{~cm}^{-1}$ region, for control heart sample (closed circles) and heart sample after three months exposure to $900 \mathrm{MHz}$ electromagnetic radiation (open circles).

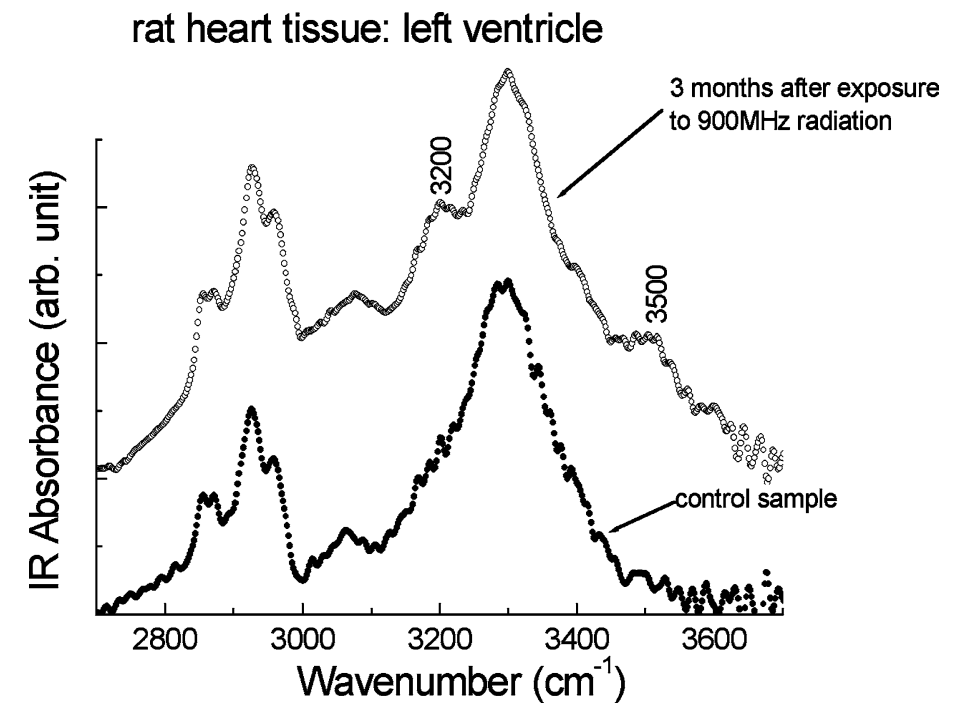

Fig. 4. FT-IR Absorbance experimental spectra, in the $2700-3700 \mathrm{~cm}^{-1}$ region, for control heart sample (closed circles) and heart sample after three months exposure to $900 \mathrm{MHz}$ electromagnetic radiation (open circles).

Finally, we focus on the bands centred, in the control sample spectrum, at $\sim 1721 \mathrm{~cm}^{-1}$ and $\sim 1737 \mathrm{~cm}^{-1}$ and corresponding to the $\mathrm{C}=\mathrm{O}$ stretching of nucleic acids and phospholipids [10], respectively. Their intensities are reduced, with respect to the control sample, in the irradiated heart. This occurrence recalls what happens in the case of an heart that suffered an infarct, where the reduced contributions of nucleic acids $\left(\sim 1721 \mathrm{~cm}^{-1}\right)$ and cellular elements such as lipids and membranes $\left(\sim 1733 \mathrm{~cm}^{-1}\right)$ are justified by the death of the myocytes and the substitution of cells with collagene caused by the infarct. 
Also in the case of left ventricle tissues, the $\mathrm{CH}_{2}$ and $\mathrm{CH}_{3}$ regions $\left(2500-3000 \mathrm{~cm}^{-1}\right)$ did not appear to be significantly altered by EMF, as it is clearly shown in Fig. 4. Nevertheless, always looking at the same figure, one immediately sees that strong variations characterize the $\mathrm{O}-\mathrm{H}$ and $\mathrm{N}-\mathrm{H}$ stretching region, i.e. $3000-3600 \mathrm{~cm}^{-1}$. When the heart is irradiated, the two contributions at $3200 \mathrm{~cm}^{-1}(\mathrm{O}-\mathrm{H}$ stretching) and $3500 \mathrm{~cm}^{-1}(\mathrm{~N}-\mathrm{H}$ stretching) are enhanced if compared to the control sample. This spectral evidence could indicate a structural rearrangement of water molecules. In this respect, since a better understanding of these complex phenomena requires a more detailed spectroscopic investigation, new FT-IR measurements are actually in progress in our laboratories.

\section{Conclusions}

With the aim to proof the FT-IR Absorbance spectroscopy to be a successful versatile technique in the biomedical filed as it has been found to be in the fields of physics, chemistry and biology, we performed a FT-IR Absorbance study of the damage caused by the exposure to low frequency ( $900 \mathrm{MHz}$ ) electromagnetic fields, on kidney and heart tissues, by estimating the spectral variations of irradiated samples with respect to a control one.

In the case of kidney tissues, the observed FT-IR spectral features confirmed, in perfect agreement with a previous morphological investigation by means of optical microscopy, that the increased perivascular connective tissue around the blood vessels suggests an increasing of the stress in the arterioral wall probably related to an hypertensive status.

As far as heart tissues are concerned, the FT-IR spectral profiles reveal an evident hypertrophic status of the cardiomyocytes which could be justified by the intercellular oedema.

On these basis we can conclude that the long exposure to EMF causes evident cellular changes and an increase of the connective tissue. A more detailed analysis from a morphological and spectroscopic point of view is in progress.

\section{References}

[1] R. Jyothi Lakshmi, V.B. Katha, C. Murali Krishna, J.G.R. Solomon, G. Ullas and P. Uma Devi, Radiation Research 157 (2002), 175.

[2] M. Jakson and H.H. Mantsch, FTIR spectroscopy in the clinical sciences, in: Advances in Infrared and Raman Spectroscopy, R.J.H. Clark and R.E. Hester, eds, Wiley, Hayden, 1996, pp. 185-215.

[3] D. Neumann, C.P. Schultz and D. Helm, in: Infrared Spectroscopy of Biomolecules, H.H. Mantsch and D. Chapman, eds, Wiley-Liss, New York, 1996, pp. 279-310.

[4] P.T.T. Wong, K. Wong and M.F.K. Fung, Appl. Spectrosc. 47 (1993), 1058.

[5] V. Crupi, S. Galli, D. Majolino, P. Migliardo, S. Pergolizzi and V. Venuti, Spectroscopy - An International Journal 16 (2002), 245.

[6] V. Crupi, D. Majolino, P. Migliardo, M.R. Mondello, M.P. Germanò and S. Pergolizzi, Vibrational Spectroscopy 25 (2001), 213.

[7] P.T. Wang, T. Kaneko, H. Tsukada, M. Nakamo, T. Nakajima and A. Sato, Arch. Toxicol. 71 (1997), 638.

[8] B. Rigas, S. Murgello, I.S. Goldman and P.T.T. Wong, Proc. Natl. Acad. Sci. USA 87 (1990), 8140.

[9] V. Crupi, unpublished data.

[10] H. Casal and H. Mantsch, Biochim. Biophys. Acta 779 (1984), 381. 


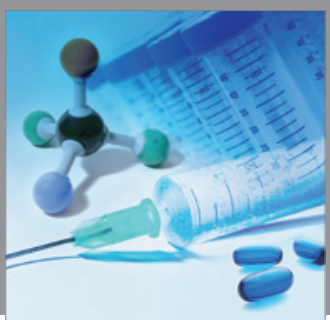

International Journal of

Medicinal Chemistry

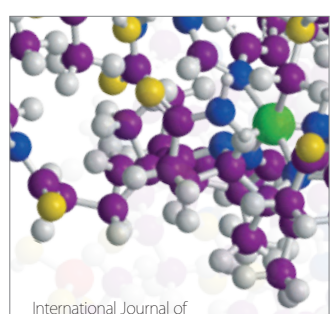

Carbohydrate Chemistry

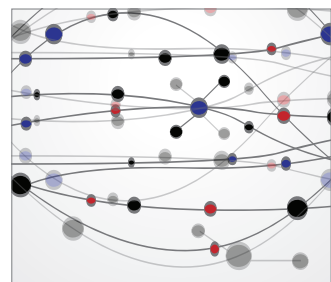

The Scientific World Journal
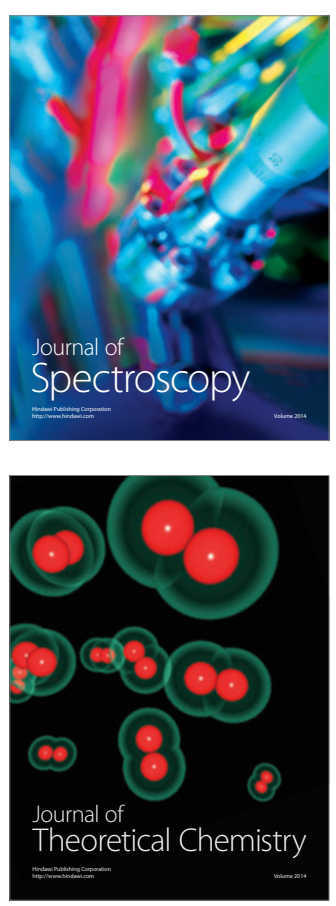
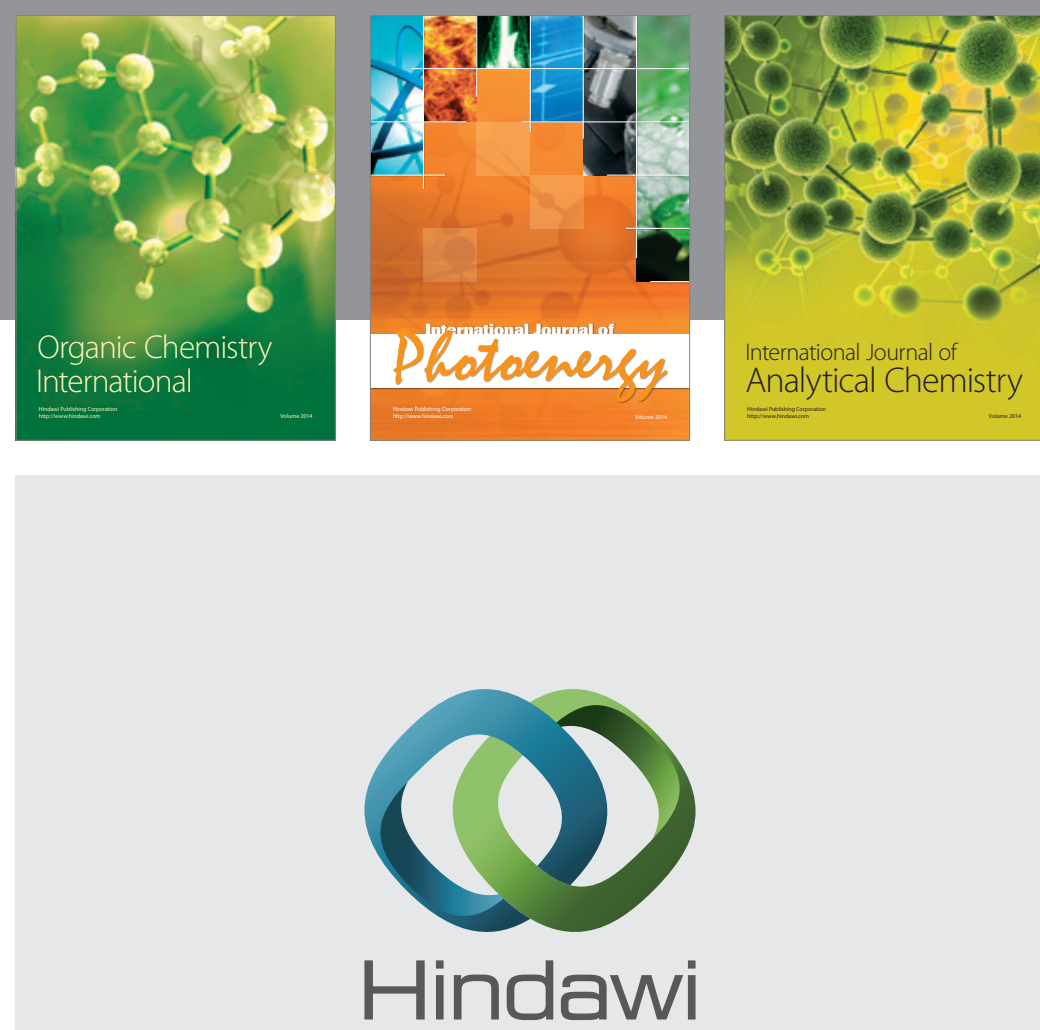

Submit your manuscripts at

http://www.hindawi.com
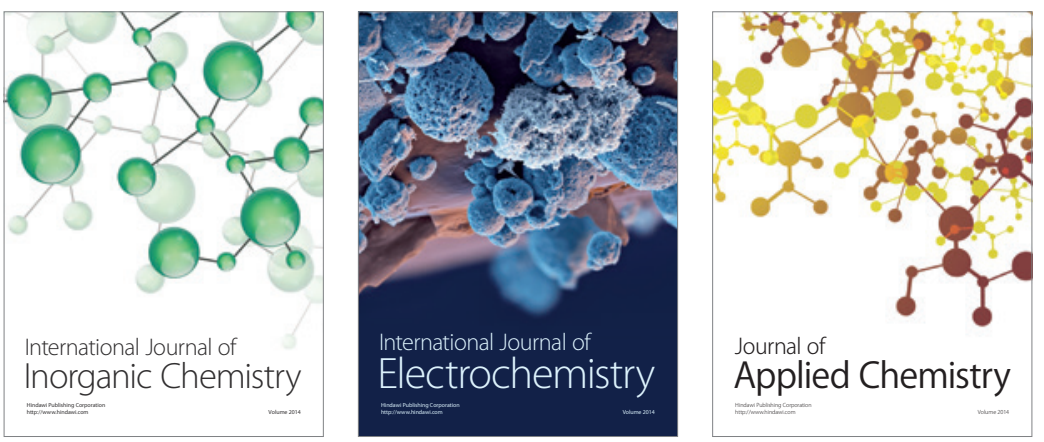

Journal of

Applied Chemistry
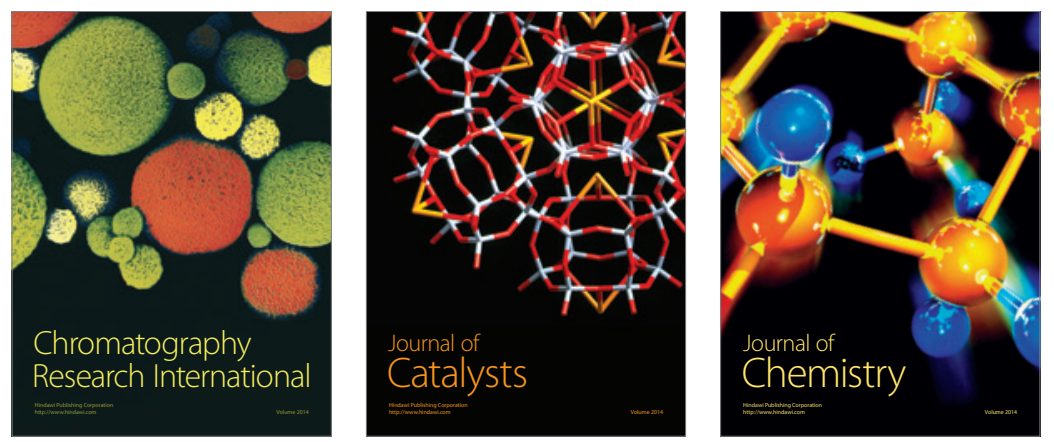
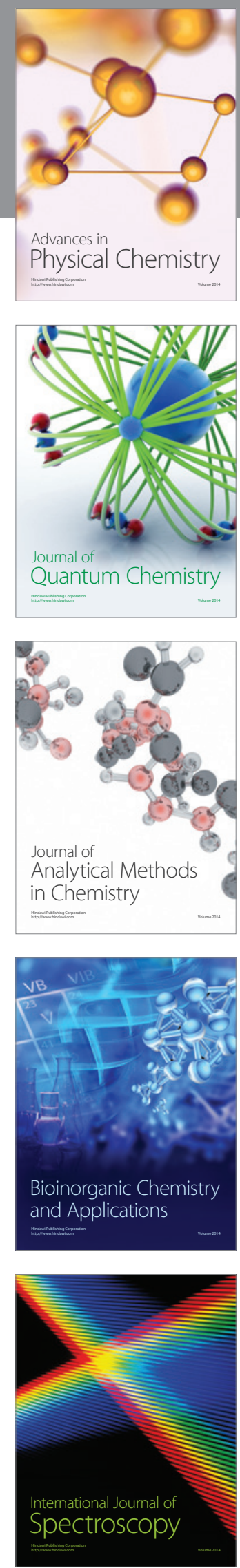